\title{
Various Kinds of Pulsed Ultraviolet Laser Micromachinings Using a Five Axis Microstage
}

\author{
Yoshiyuki Kawamura, Akihiro Kai and Keita Yoshii \\ Department of Intelligent Mechanical Engineering, Faculty of Engineering, Fukuoka Institute of Technology \\ 3-30-1, Wajirohigashi, Higashiku, Fukuoka, 811-0295, Japan \\ E-mail: kawamura@fit.ac.jp
}

\begin{abstract}
In this paper, various kinds of laser micromachinings using a pulsed ultra violet laser are reported. Procedures for three dimensional axis alignments between the laser beam axis and the axes of the microstage were established, which determines the accuracy of machining. Various types of micromachining are described ranging from fundamental machining of "cutting", "grinding" and "engraving" to complex structures such as micro globe and rotating micro windmill.
\end{abstract}

DOI:10.2961/jlmn.2010.02.0012

Key Words: Micromachining, Five-axis, Ultraviolet laser, Laser ablation

\section{Introduction}

Since the beginning of 1980 s, pulsed ultraviolet lasers including Excimer lasers, have been applied to various types of laser micro fabrications [1-5]. Among them, the optical prototyping method has been developed to become the most popular method for micromachining [6], after the laser beams were introduced as the light source [7-9]. Although this method is widely used in the industry, it has a disadvantage that the material is limited to photopolymers.

The laser lathe method was invented for the machining of three dimensional micro structures in 1995 [10]. It can be used for various kinds of materials, such as plastics, metals, ceramics and even diamonds, provided that the laser beam is absorbed by these materials. This method was improved to be the five axis laser micro machining, and a simple 3D micro patterning of a small sphere was performed [11]. Recently, self controlling of the machining depth was successfully demonstrated to fabricate $2.5 \mathrm{D}$ micro structures (arrays of micro-pyramids) making use of the ablation threshold of the laser beam [12].

In this paper, various kinds of laser micromachining are reported, as well as the improved alignment method between the laser beam and the axes of the microstage. The authors think that this method can be one of the effective 3D laser micro machining methods, complementing disadvantages of the optical prototyping micromachining method.

\section{Five axis laser micromachining system}

\subsection{Concepts of 3-D laser micro machining}

As is shown in Fig.1, when the laser beam is scanned perpendicular to the laser beam axis, the cutting plane can be generated. In solid materials, the laser beam does not diverge due to the optical guiding by the surrounding wall of the material. Therefore relatively flat cutting plane is expected to be generated.

If the laser beam irradiates the material surface parallel to the finished surface, and is scanned perpendicularly to the laser beam axis, the smooth surface can be obtained.

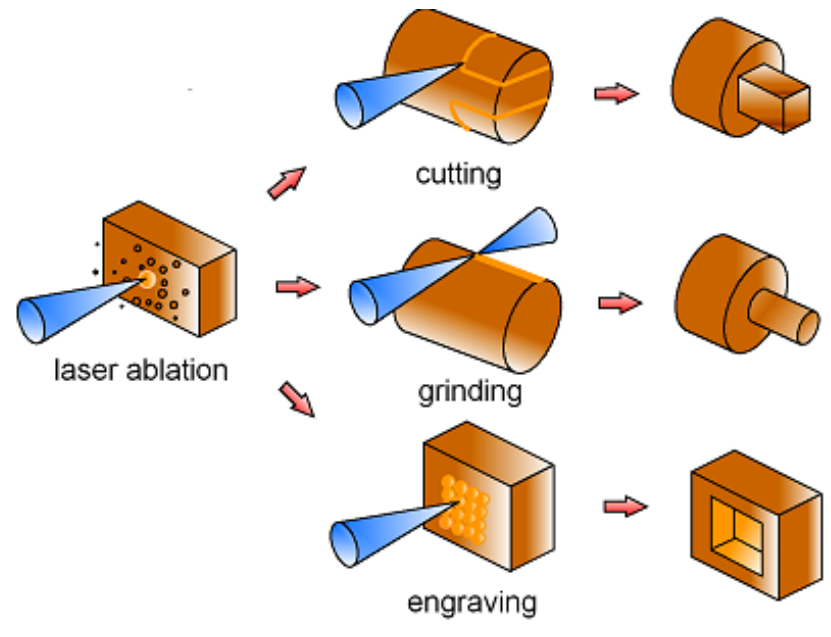

Fig. 1 Various types of laser machining.

Additionally, if the material is rotated around the axis perpendicular to the laser beam axis, cylindrical shape can be generated after sufficient time is taken.

When the laser beam is scanned two dimensionally perpendicular to the laser beam axis, an engraved volume is created.

\subsection{Experimental setup}

Fig. 2 shows the experimental setup for the five axis laser micro machining. The fourth harmonic beam of a $Q$ switched Nd-YAG laser $(266 \mathrm{~nm})$ is focused onto a pinhole with the diameter of $0.8 \mathrm{~mm}$. The image of the pinhole is relayed on to the surface of the materials using the telescopic optics with the magnification ratio of $1 / 50$. Therefore, the focusing spot size is calculated to be $15 \mu \mathrm{m}$ or less. The energy, the pulse duration and the repetition rate are about 1 $\mathrm{mJ}, 10 \mathrm{~ns}$ and $30 \mathrm{~Hz}$, respectively.

The five axis microstage and the laser are driven automatically by a TTL board, which is controlled by a personal computer (PC). Generally, it takes several millions 


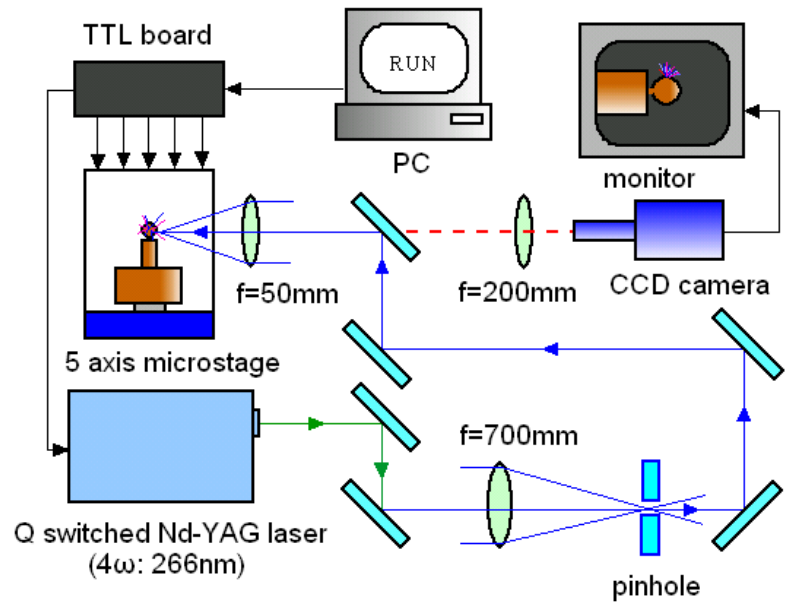

Fig. 2 Five axis laser micro machining system.

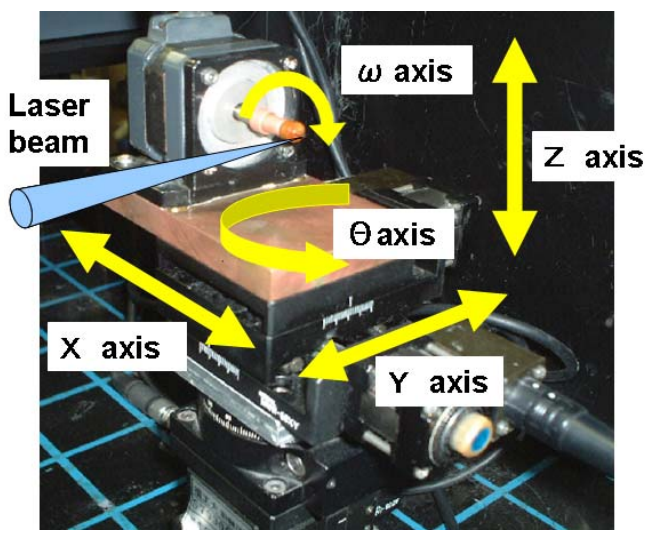

Fig. 3 Five axis micro stage.

shots to fabricate a micro structure.

For the monitoring of the machining conditions, a CCD video camera takes the images of the work piece through the telescopic optics with the magnification of 5 times.

Fig.3 shows the five axis microstage. The stepping distance to the $X, Y$ and $Z$ directions is $0.5 \mu \mathrm{m}$. The work piece is attached on the top of the $\omega$ axis, which is parallel to the $X$ axis. $\theta$ is the rotation around the $Z$ axis.

Generally, the stepping motors show small vibration at the low rotation speed. Therefore "fine step" type was used for $\omega$ rotation to avoid this vibration.

\section{Fabrication of fundamental shapes \\ 3.1 Cubic shape}

Fig. 4 shows a micro cube fabricated using the cutting method from bulk polyimide material. White scale bar represents $100 \mu \mathrm{m}$. It is interesting that flat surfaces are machined on the cube in spite of using the conically focusing laser beam.

A long hole in the center was machined using the cutting method. In order to separate the cylinder from the bulk, a rectangular side slit was fabricated using the engraving method.

\subsection{Cylindrical shape}

Fig. 5 (a) shows a procedure to fabricate a cylinder from a bulk material. The laser beam is scanned parallel to the $X$

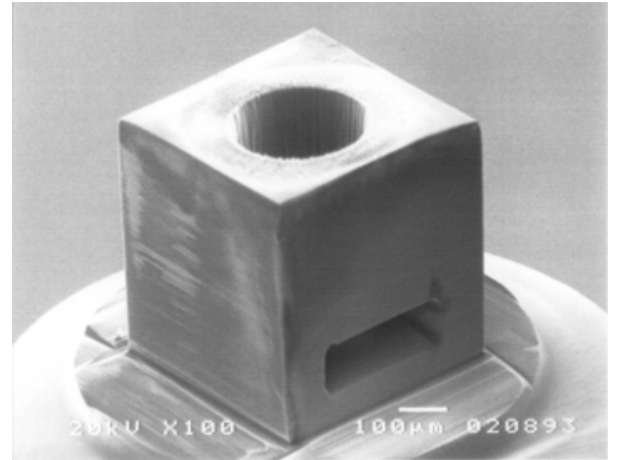

Fig. 4 Micro cubic having a hole in it.

axis at the same radial position decreasing the radius gradually. The procedure is repeated continuously, until the radius of the desired size is achieved.

It is also possible to skip the scanning process to the radial direction and adjust the laser beam at the desired value from the beginning of the machining. The machining must be continued, until the ablation on the surface stops. It can be confirmed by the disappearance of the formation of the plasma or the generation of machining sound.

Fig. 5 (b) shows a typical micro cylinder with the diameter of $200 \mu \mathrm{m}$. The material is polyimide, which has good machinability by UV laser ablation.

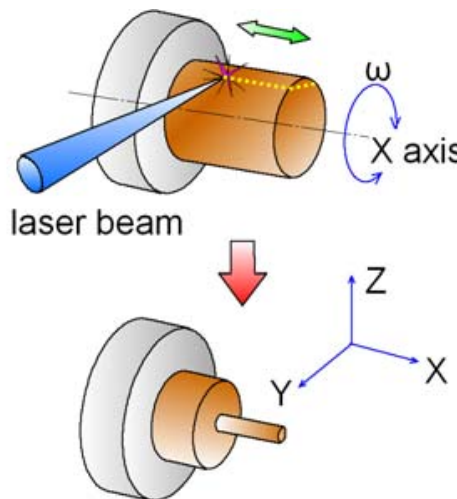

(a)

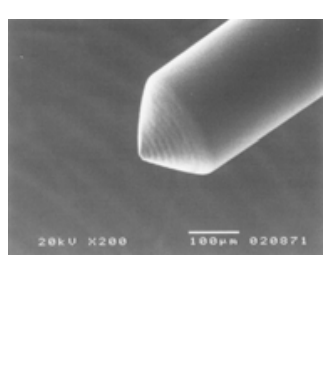

(b)
Fig. 5 Fabrication of micro cylinders. (a): Machining process of a micro cylinder. (b): Typical micro cylinder with the diameter of $200 \mu \mathrm{m}$ machined by this method.

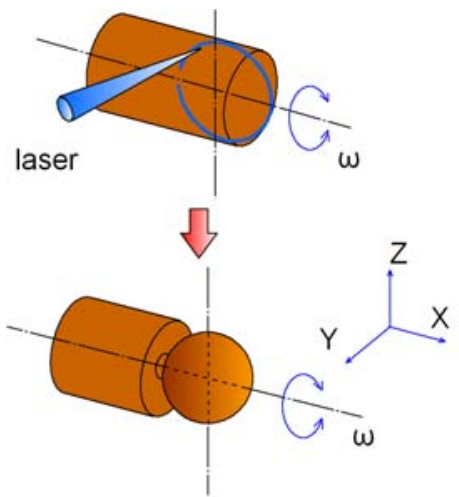

(a)

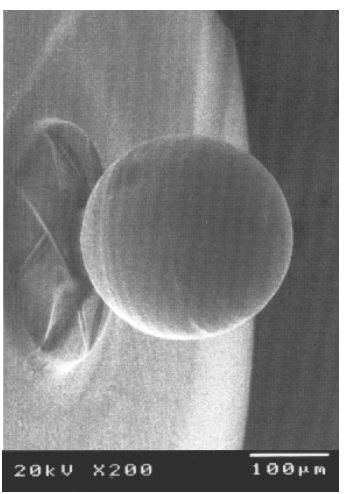

(b)
Fig. 6 Fabrication of micro sphere. (a): Machining process of the micro sphere. (b): Typical micro sphere with the diameter of $200 \mu \mathrm{m}$. 


\subsection{Sphere}

Fig. 6 (a) shows a procedure to fabricate a sphere from the cylinder. The diameter of the cylinder is slightly larger than that of the sphere.

The laser beam was scanned along the circular trajectory except of the connecting region between the sphere and the bulk material, until the finished spherical shape was obtained.

Fig. 6 (b) shows a typical micro sphere with the diameter of $200 \mu \mathrm{m}$ machined by this method. The material is also polyimide.

\section{Axes alignments}

When the cylinder is fabricated as shown in Fig. 5, the $X$ axis is automatically determined at the central position between the top surface and the bottom surface of the cylinder.

When the sphere is fabricated as shown in Fig. 6, the origin of the $X-Z$ surface is automatically determined at the center of the sphere.

For the precise $3 \mathrm{D}$ micro machining, the rotational axis of $\theta$, which is the mechanical rotational axis of the micro stage, must be aligned on the $Z$ axis.

Fig. 7 shows the ideal positions of the laser beam and the $X-Y-Z$ coordinate system. This condition can be realized by adjusting the mechanical rotational axis $\theta$ to the $Z$ axis through a trial and error process monitoring the image of the micro sphere in the monitor screen of a CCD camera. Namely, the position of the $\theta$ axis is moved along the $X$ axis, so that the microsphere does not move in the monitor screen, while the work rotates 180 degrees around the $\theta$ axis. The accuracy of the alignments was estimated to be about $3 \mu \mathrm{m}$ judging from the magnification of the optics for the monitor and the number of pixels of the CCD camera.

\section{Five-axis laser micro fabrications \\ 5.1 Fabrication of a sharp tip}

Fig. 8 (a) shows a micro conical shape. The focusing spot size of the laser beam was about $15 \mu \mathrm{m}$. The machining procedure is similar to that for the cylinder, except that the direction of the scanning is not parallel to the $X$-axis, but is tilted with a half apex angle of the conical shape. Fig.8 (b) shows a magnified of tip of the conical shape. In spite of the large diameter of the laser beam $(15 \mu \mathrm{m})$, a sharp tip was obtained, whose radius of curvature is much less than the radius of the laser beam.

\subsection{Fabrication of complex structure (micro globe)}

Fig. 9 shows a mounting part of a micro globe, which

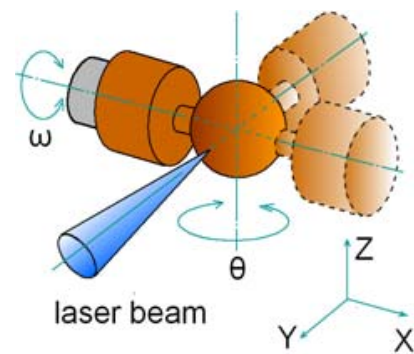

Fig. 7 Axis alignment between $\theta$ axis and laser beam axis

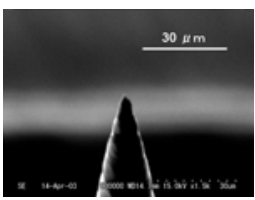

(a)

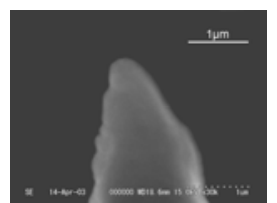

(b)
Fig. 8 Fabrication of a micro conical shape. (a): Micro conical shape. (b): Magnifications of the top of the micro conical shape.
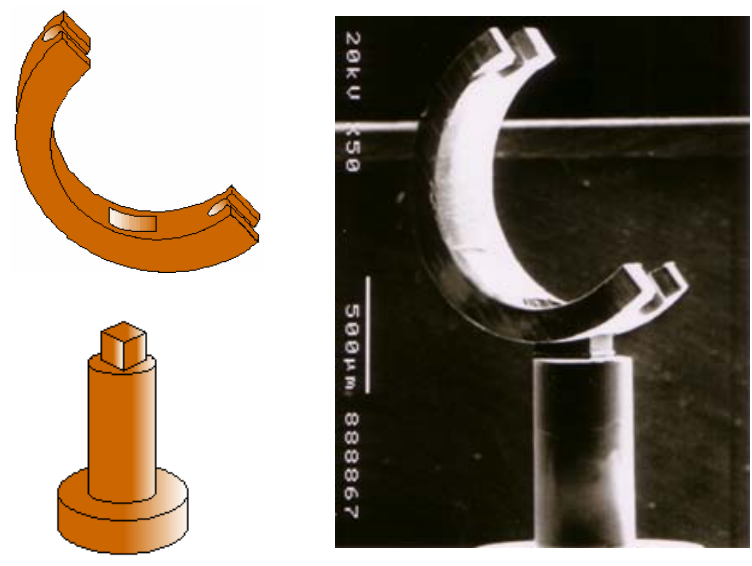

Fig. 9 Mounting part of the micro globe.

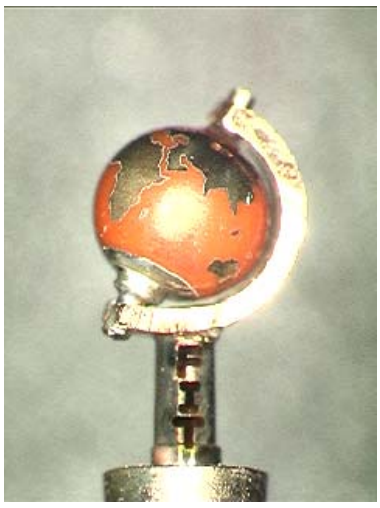

Fig. 10 Micro globe mounted on the bearing part

consists of a semi-circular bearing part for supporting the micro globe and a base. The former was machined using a cutting method, while the latter was machined using the "laser lathe" method, except for a square connecting part between these two. We don't have a suitable assembling method which can be applied for these micro parts, therefore they were assembled manually.

Fig. 10 shows the micro globe mounted on the bearing part. The diameter of the globe is $1 \mathrm{~mm}$. The map on the globe was fabricated by ablating a gold thin film evaporated on the surface of the globe.

\subsection{Fabrication of a micro windmill (moving device) 5.3.1 Fabrication}

Fig. 11(a) shows a micro windmill with the base. The diameter of the windmill is $800 \mu \mathrm{m}$. The diameter of the 
shaft is $160 \mu \mathrm{m}$. The whole machining process is conducted automatically by a program. Generally, it takes more than 10 hours, therefore machining was performed from the evening to the morning of the next day.

The windmill was separated from the base, and it was put into the hole of the air bearing, whose inner diameter is slightly larger than the outer diameter of the windmill shaft.

Fig. 11(b) shows a magnification of the blades of the micro windmill. Periodical machined grooves of $10 \mu \mathrm{m}$ pitch can be seen.

Fig. 12 shows a hole and a flat surface of the air bearing for the micro windmill. The diameter of the hole is $240 \mu \mathrm{m}$. The hole was machined by the cutting method and the flat surface was finished by the grinding method. The material was polyimide.

\subsubsection{Rotation of the windmill}

In order to evaluate the mechanical characteristics of the micro windmill, we tried to rotate it in a high speed air flow, which was produced by expanding the air of the standard condition (the air in the room) into a vacuum chamber through a small throat as shown in Fig. 13. The flow was considered to be accelerated up to the supersonic speed, because it was the "choked flow".

The windmill was separated from the basement to mount into the air bearing. The back pressure of the air bearing was adjusted by controlling the flow rate between the rotating shaft and the cylindrical wall surrounding it by use of a needle valve.

The rotational speed of the windmill was optically measured by transmitting a long focused diode laser beam between the blades and measuring the frequency of the transmitted light by a FFT analyzer. The measured frequency was divided by the number of the blades, and the speed was determined to be about $3.6 \times 10^{5} \mathrm{rpm}$.

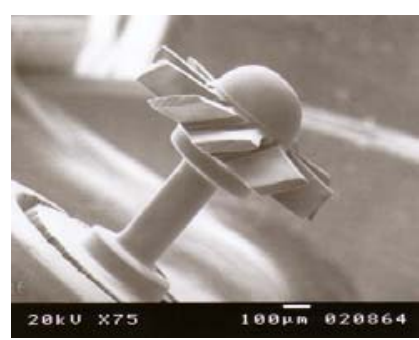

(a)

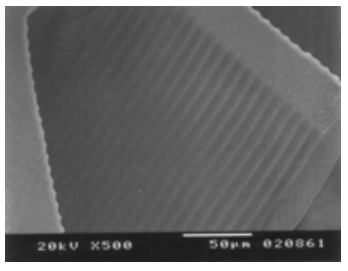

(b)
Fig. 11 Micro windmill (a): Micro windmill with a basement before being separated. (b): Machined grooves on the blade of the micro windmill. Pitch is $10 \mu \mathrm{m}$.
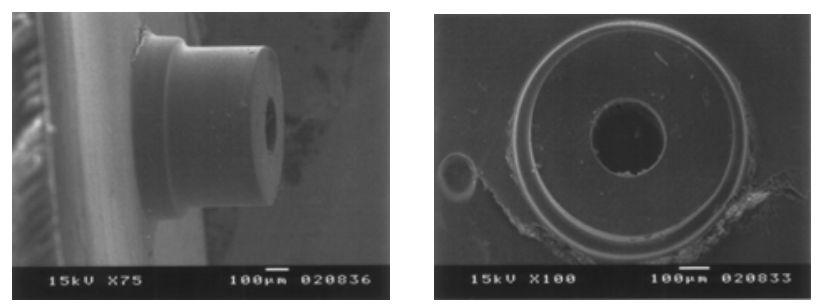

Fig. 12 Hole and flat surface of the air bearing for the rotation of the micro windmill.

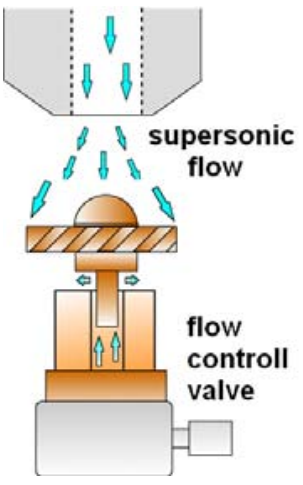

Fig. 13 Rotation experiment of the micro windmill with the air bearing in the supersonic flow.

\subsection{Fabrication of a diamond micro drill (hard material) 5.4.1 Fabrication of a blade}

Fig. 14 shows a conceptual scheme to fabricate a sharp edge on the outlet side of a laser beam stream. When a hole is fabricated by a laser beam, sharp edge can be obtained at the corner of the outlet of the hole (down stream side), while the corner at the inlet (upstream side) becomes rounded.

This phenomenon is also seen in the field of a precision machining using a focused ion beam [13]. Fig. 15 (a) shows a typical sharp edge fabricated at the outlet of the hole using the method described above. Fig. 15 (b) shows the rounded edge obtained at the inlet of the hole. Fig. 16 shows a pair of blades of a micro drill fabricated using five-axis laser micro machining system. Materials are all industrial diamonds. The formation of this sharp edge belongs to the category of grinding laser micro machining.
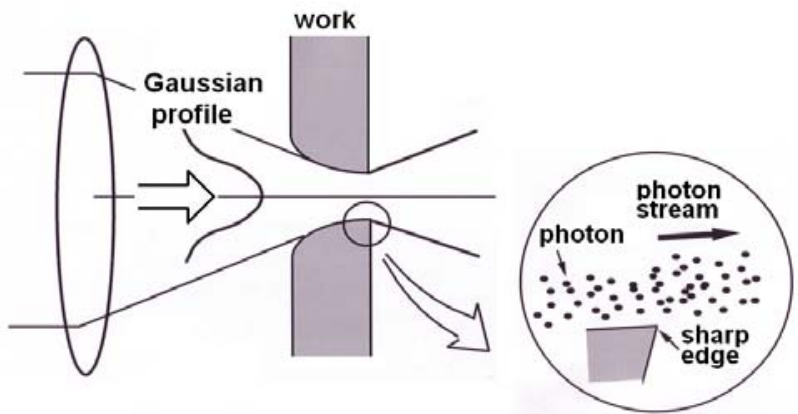

Fig. 14 Sharp edge formation making use of a stream of photons.

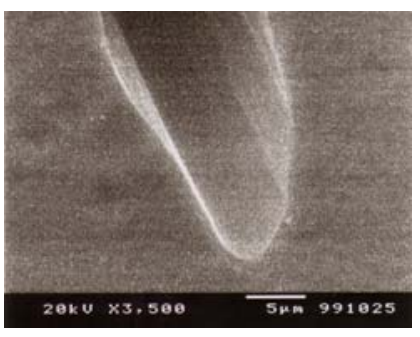

(a)

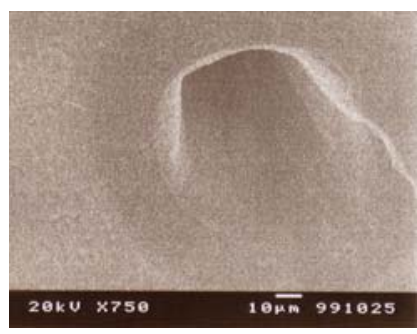

(b)
Fig. 15 (a): Outlet side of the hole. Sharp edge is machined. White scale bar represents $5 \mu \mathrm{m}$. (b): Inlet side of the hole. The edge is not sharp but rounded. White scale bar represents $10 \mu \mathrm{m}$. 


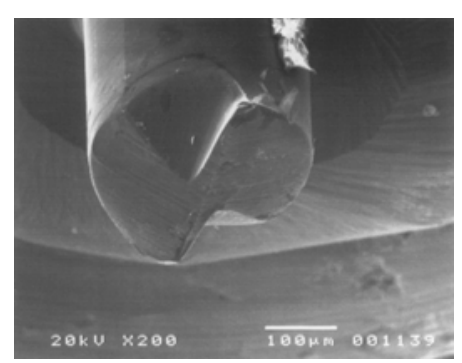

Fig. 16 Micro drill fabricated from an industrial diamond. White bar represents $100 \mu \mathrm{m}$.

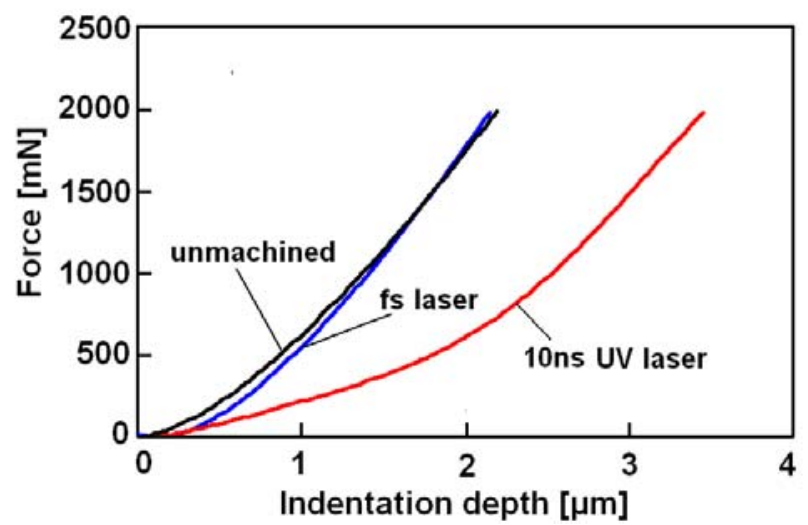

Fig. 17 Vickers microhardness test of the laser machined surface of a natural diamond.

\subsubsection{Degradation of the surface hardness}

The red line in Fig. 17 shows the measurement of Vickers microhardness test obtained for the surface of a natural diamond machined by the laser grinding. The slope is considered to be proportional to the hardness. Up to a depth of about 2 to $3 \mu \mathrm{m}$, the degradation of the hardness can be observed. This can be considered to be due to the heating by the laser beam.

Considering that the thermal diffusion in the laser pulse duration is induced by the temperature gradient generated by the laser irradiation and the diffusing heat compensate this temperature gradient, the thermal diffusion length $l$ in the laser pulse duration $\tau$ can be estimated to be $l=\sqrt{k \tau / c \rho}$, where $\kappa, c$ and $\rho$ are the coefficient of thermal conductivity, the specific heat capacity and the density of the diamond, respectively. Substituting $\kappa=2000 \mathrm{~W} / \mathrm{mK}, \tau=1 \times 10^{-8} \mathrm{~s}, c$ $=513 \mathrm{~J} / \mathrm{kgK}$ and $\rho=3.5 \times 10^{3} \mathrm{~kg} / \mathrm{m}^{3}, l$ is calculated to be $3.3 \mu \mathrm{m}$, which coincides approximately with the measured value.

For the comparison, the same machining was performed using a femtosecond laser. The corresponding microhardness measurements are shown by a blue line. Judging from the slope of the blue line, the thermally degraded region was much smaller than that of $10 \mathrm{~ns}$ UV laser. Black line represents the measurement for the unmachined surface of the natural diamond.

These results show the limitation of applying the long pulse laser, such as $10 \mathrm{~ns}$ UV laser, to the fabrication of hard diamond micro blades, and the advantages of the femtosecond laser in machining diamond.

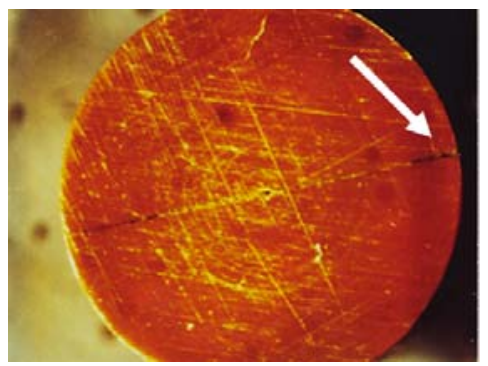

Fig. 18 Cross section of an optical guided long hole machined in the cylinder of a polyimide material. White arrow shows the inlet of the laser beam.

\section{Discussion}

The laser machining has more advantages in the case of micro machining than in the case of normal size machining. One of the advantages is the relatively long Rayleigh length of the focusing region.

For example, the Rayleigh length is calculated to be about $100 \mu \mathrm{m}$, when the NA=0.05 and the wavelength is $0.25 \mu \mathrm{m}$. Even though the laser beam is focused having an angle of the focusing corn, relatively long machining region can be obtained, if the work is small, because the diameter of the focusing spot size $16 \mu \mathrm{m}$ is almost the same size in the Rayleigh region.

Another advantage is the optically guiding of the laser beam, which can be observed in the bulk materials, and by which the diameter of the laser beam is guided for long distance keeping the spot size constant.

Fig. 18 shows the cross section of an optical guided long hole machined in a polyimide cylinder material. Black straight line crossing the cylinder is the cross section of the drilled hole. The diameter of the cylinder is $6 \mathrm{~mm}$, and the diameter of the drilled hole is estimated to be about $30 \mu \mathrm{m}$. This sample was prepared by cutting out the cross section and polishing it after the laser drilling.

\section{Conclusions}

Procedures for three dimensional axis alignment between the laser beam axis and the axes of the microstage were established, which determines the accuracy of machining. Various types of micromachining are described ranging from fundamental machining of "cutting", "grinding" and "engraving" to complex machining of intricate parts such as micro globe and rotating micro windmill. The rotation speed was measured to be about $3.6 \times 10^{5} \mathrm{rpm}$.

The method for generating a sharp edge is described and demonstrated. The limitation of applying the long pulse laser, such as $10 \mathrm{~ns}$ UV lasers to the fabrication of hard diamond micro blades was described, and the possibility for applying the femtosecond laser to fabricate sharp and hard diamond blades was also described.

\section{References}

[1] Y. Kawamura, K. Toyoda and S. Namba, Review of Laser Engineering, 8, 941 (1980) (in Japanese).

[2] Y. Kawamura, K. Toyoda, and S. Namba: Appl. Phys. Lett.,40, 374 (1982). 
JLMN-Journal of Laser Micro/Nanoengineering Vol. 5, No. 2, 2010

[3] Y. Kawamura, K. Toyoda, and S. Namba: J. Appl. Phys., 53, 6489 (1982).

[4] P. E. Dyer, Appl. Phys. A77, 167 (2003).

[5] K. Sugioka, S. Wada, H. Tashiro, K. Toyoda, Y. Ohnuma, and A. Nakamura, Appl. Phys. Lett. 67, 2789 (1995).

[6] H. Kodama, Review of Science Instrument, 52, 1770 (1981).

[7] T. Nakai and Y. Marutani, Rev. of Laser Engineering, 16, 14 (1988) (in Japanese).

[8] T. Nakai and Y. Marutani, Applied Optics, 33, 25
(1992).

[9] S. Maruo and H. Inoue, Applied Physics Letters, 89,144101(2006).

[10] Y. Kawamura and K. Toyada, Review of Laser Engineering, 23, 676 (1995) (in Japanese).

[11] Y. Kawamura and A. Takenaka, Review of Laser Engineering, 27, 45 (1999) (in Japanese).

[12] T. Yamaguchi and T. Semba, Transactions of the JSME, Ser. C, 73, 220 (2007) (in Japanese).

[13] Y. N. Picard, D. P. Adams, M. J. Vasile and M.B. Ritchey, Precision Engineering, 27, 59 (2003).

(Received: April 8, 2010, Accepted: May 22, 2010) 